\title{
Concordance rates in twins for anencephaly
}

\author{
WILLIAM H JAMES \\ From the Galton Laboratory, University College London, Wolfson House, \\ 4 Stephenson Way, London NW1 $2 \mathrm{HE}$
}

SUMMARY New estimates are offered of the concordance rates in twins for anencephaly. In MZ pairs, the percentage which are concordant is about 7. This is significantly higher than (about three or four times) the value expected on the assumption that concordance is accounted for by raised recurrence rates within sibships. In DZ pairs, the data are less numerous and the percentage concordant (about 2 to 5 ) does not so far throw much suspicion on this hypothesis in regard to them.

Speculation about the aetiology of anencephaly has been based on, inter alia, the supposed concordance rates in twins. I have suggested that the concordance rate is high in monozygotic (MZ) pairs even bearing in mind (1) the raised recurrence rates within sibships, and (2) the hypothesised higher incidence in MZ pairs. ${ }^{12}$ Since those papers were prepared, the data have been reviewed ${ }^{3}$ and two further large samples presented. ${ }^{5}$ Lorber and Rogers ${ }^{3}$ and Imaizumi $^{5}$ both offer estimates of concordance which are based on unconventional methods of estimating the numbers of MZ pairs. Accordingly I should like to offer revised estimates.

Table 1 summarises the data on anencephalic twins ascertained in series.

\section{Dizygotic pairs}

It will be seen from table 1 that of 138 oppositesexed pairs with anencephaly five were concordant. It is worth estimating the number expected on the assumption that the concordance rate in such pairs is based solely on the raised recurrence rates within sibships. Data on the recurrence rate in anencephaly

TABLE 1 Numbers of pairs of twins concordant and discordant for anencephaly ascertained in series

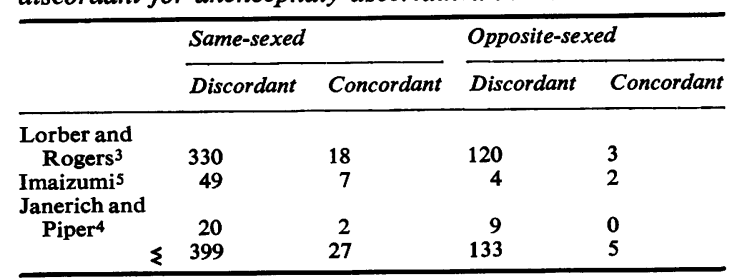

Pairs in which one twin has anencephaly and the other spina bifida are counted as discordant.

Received for publication 25 September 1979 have been usefully reviewed by Fedrick. ${ }^{6}$ She pooled data from 17 studies (twin data from 12 of these are included in table 1); she found that of 5373 sibs of anencephalic probands $135(2 \cdot 5 \%)$ were themselves anencephalic. This value $(2 \cdot 5 \%)$ will be taken as representative of the populations from which the samples of twins were drawn. Where ascertainment is complete (as may be presumed in series of cases), $r \bumpeq 2 c$ where $r$ is the recurrence rate and $c$ the concordance rate. From which the expected number of concordant cases among 138 affected pairs would be

$$
138 \times \frac{2 \cdot 5}{100} \times \frac{1}{2}=1 \cdot 7
$$

The Poisson probability of five or more observations when 1.7 are expected is about 0.03 . However, the question of whether concordant pairs were disproportionately likely to be reported in Imaizumi's material is raised by the fact that the sexes of nearly half of her pairs were unknown. If her data are omitted from consideration, the analogous Poisson probability for the remaining three cases is about 0.23 . Thus, there is a suspicion, but not more, that the concordance rate in $\mathrm{DZ}$ twins is higher than can be accounted for by the raised recurrence rates within sibships.

\section{Monozygotic pairs}

Lorber and Rogers ${ }^{3}$ and Imaizumi ${ }^{5}$ estimated the concordance rates in $\mathrm{MZ}$ twins as follows.

(1) They assumed that the concordance rate in same-sexed DZ pairs is the same as in opposite-sexed DZ pairs, and

(2) they assumed that among same-sexed pairs a fixed percentage are $\mathbf{M Z}$ (Lorber and Rogers $40 \%$ : Imaizumi $30 \%$ ).

Both these points may be commented on. 
In a sex specific condition, one would not expect the concordance rate in same-sexed pairs to be the same as that in opposite-sexed pairs, even if the incidences of the condition in $\mathrm{MZ}$ and $\mathrm{DZ}$ twin pairs were the same. However, it is shown in the Appendix that though the sex specificity of anencephaly has some effect on the expected concordance rates, it is not, by itself, substantial.

In a large sample of twin pairs, the proportion which are MZ may be estimated using Weinberg's Differential Rule. I have suggested ${ }^{7}$ that there is a slight flaw in this. However, this would only lead to seriously biased estimates of the proportion of $\mathbf{M Z}$ pairs where the true proportion of $\mathrm{MZ}$ pairs is low and this does not apply to the present data.

The assumption underlying Weinberg's Rule is that, among $\mathrm{DZ}$ pairs, the number of same-sexed pairs is equal to the number of opposite-sexed pairs. There seems no reason for supposing this to be far from true in regard to anencephalic pairs (that is, those in which one or both are anencephalic). This is so even though, as noted above, concordance rates would be expected to differ slightly between same-sexed and opposite-sexed pairs.

If we apply these considerations to the data in table 1 it would be reasonable to estimate that, among the same-sexed pairs, there are about 138 DZ pairs of which say six (=5 $\times 5 / 4)$ are concordant. This leaves $288 \mathrm{MZ}$ pairs of which $21(7 \%)$ are concordant. If one accepts that the incidence rate in $\mathrm{MZ}$ twins is $1 \frac{2}{3}$ higher than in other births ${ }^{1}$ (and that the recurrence rate is proportionately raised in MZ twin sibships), then the number of concordant $\mathrm{MZ}$ pairs expected on the assumption that the concordance rate is based on the raised recurrence rate within sibships is

$$
288 \times 0.025 \times 1 \frac{2}{3} \times \frac{1}{2}=6 .
$$

So it seems that the concordance rate in MZ twins is perhaps three or four times higher than can be accounted for by the raised recurrence rate within sibships.

I am supported by the National Fund for Research into Crippling Diseases.

\section{Appendix Effect of sex specificity on expected con-} cordance rates.

Referring to the data in table 2 , the concordance rate for same-sexed pairs is

$$
\frac{p^{2}+n^{2} p^{2}}{p^{2}+n^{2} p^{2}+2 p(1-p)+2 n p(1-n p)}
$$

and the concordance rate for opposite-sexed pairs is

$$
\frac{n p^{2}}{n p^{2}+p(1-n p)+n p(1-p)} \text {. }
$$

If $p$ is small, then the ratio of these rates may be shown to be approximately

$$
\left(1+n^{2}\right) / 2 n \text {. }
$$

Most of the twin pairs in series have been ascertained in areas of moderate or low incidence (USA, Holland, Canada, and Australia): the point may be seen by referring to the tables of Lorber and Rogers (privately circulated in supplement to their 1977 paper). In such areas, females do not outnumber males by a ratio of more than $2: 1$. In other words, n may be taken to be 2 or less for those samples, and the ratio of the rates to be $5: 4$ or less. For those samples drawn from high incidence populations, one might take $n=3$ and the ratio $10: 6$, but it seems reasonable to accept $5: 4$ as the ratio for the total sample. So for all the data summarised here, one might suggest that among concordant DZ pairs, same-sexed pairs would outnumber oppositesexed pairs in the ratio of about $5: 4$.

TABLE 2 Relative frequencies of affected $M M, M F$, and $F F$ pairs

\begin{tabular}{llll}
\hline & No of affected & & \\
\cline { 2 - 4 } Twin type & 0 & 1 & 2 \\
\hline MM & $(1-\mathrm{p})^{2}$ & $2 \mathrm{p}(1-\mathrm{p})$ & $\mathrm{p}^{2}$ \\
FF & $(1-\mathrm{np})^{2}$ & $2 \mathrm{np}(1-\mathrm{np})$ & $\mathrm{n}^{2} \mathrm{p}^{2}$ \\
MF & $(1-\mathrm{p})(1-\mathrm{np})$ & $\mathrm{p}(1-\mathrm{np})+\mathrm{np}(1-\mathrm{p})$ & $\mathrm{np}^{2}$ \\
\hline
\end{tabular}

This table gives the relative frequencies of MM, MF, and FF twin pairs expected with 0,1 , and 2 affected if (1) the probability of a male being affected is $p ;(2)$ the probability of a female being affected is np; and (3) the probability of one twin being affected is independent of that of the other.

\section{References}

1 James WH. Twinning and anencephaly. Ann Hum Biol $1976 ; 3: 401-9$.

2 James WH. Recurrence rates in sibships and concordance rates in twins for anencephaly. Ann Hum Biol 1978;5: 79-81.

3 Lorber J, Rogers SC. Spina bifida cystica and anencephalus in twins. $Z$ Kinderchir 1977;22:565-71.

4 Janerich DT, Piper J. Shifting genetic patterns in anencephaly and spina bifida. J Med Genet 1978;15:101-5.

5 Imaizumi Y. Concordance and discordance of anencephaly in 109 twin pairs in Japan. Jpn J Hum Genet 1978; 23:389-93.

- Fedrick J. Anencephalus in the Oxford Record Linkage Study area. Dev Med Child Neurol 1976;18:643-56.

7 James WH. The possibility of a flaw underlying Weinberg's Differential Rule. Ann Hum Genet 1976; 40:197-9.

Requests for reprints to Dr W H James, Department of Human Genetics and Biometry, The Galton $\frac{?}{\mathbb{Q}}$ Laboratory, University College London, Wolfson $\unrhd$ House, 4 Stephenson Way, London NW1 2HE. 